\title{
Collective Effects in Second-Harmonic Generation from Plasmonic Oligomers
}

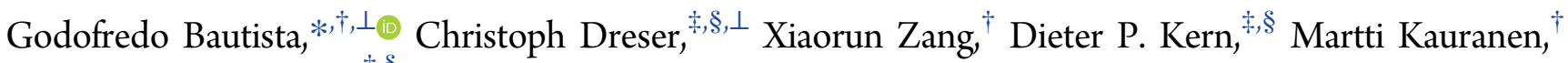 \\ and Monika Fleischer*, ${ }^{*}, \S$
}

\author{
${ }^{\dagger}$ Laboratory of Photonics, Tampere University of Technology, Korkeakoulunkatu 3, 33720 Tampere, Finland \\ ${ }^{\ddagger}$ Institute for Applied Physics, University of Tübingen, Auf der Morgenstelle 10, 72076 Tübingen, Germany \\ ${ }^{\S}$ Center for Light-Matter-Interaction, Sensors and Analytics LISA+, University of Tübingen, Auf der Morgenstelle 15,72076 \\ Tübingen, Germany
}

\section{Supporting Information}

ABSTRACT: We investigate collective effects in plasmonic oligomers of different symmetries using second-harmonic generation (SHG) microscopy with cylindrical vector beams (CVBs). The oligomers consist of gold nanorods that have a longitudinal plasmon resonance close to the fundamental wavelength that is used for SHG excitation and whose long axes are arranged locally such that they follow the distribution of the transverse component of the electric field of radially or azimuthally polarized CVBs in the

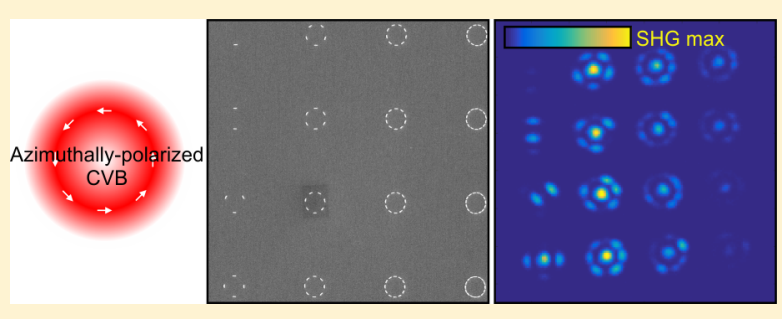
focal plane. We observe that SHG from such rotationally symmetric oligomers is strongly modified by the interplay between the polarization properties of the CVB and interparticle coupling. We find that the oligomers with radially oriented nanorods exhibit small coupling effects. In contrast, we find that the oligomers with azimuthally oriented nanorods exhibit large coupling effects that lead to silencing of SHG from the whole structure. Our experimental results are in very good agreement with numerical calculations based on the boundary element method. The work describes a new route for studying coupling effects in complex arrangements of nano-objects and thereby for tailoring the efficiency of nonlinear optical effects in such structures.

KEYWORDS: Cylindrical vector beams, plasmonic oligomers, second-harmonic generation microscopy, electron beam lithography, boundary element method

$\mathrm{N}$ onlinear plasmonics has been receiving widespread attention in recent years. ${ }^{1}$ Here, metal nanoparticles, whose optical response is governed by localized surface plasmon resonances, are used to significantly enhance nonlinear optical effects. To date, the majority of the work on nonlinear plasmonics has been based on arrays of nanoparticles, ${ }^{2,3}$ individual nanoparticles, ${ }^{4,5}$ pairs of nanoparticles, ${ }^{6-8}$ or nanoparticle systems which consist of metal and dielectric components. ' While many previous works highlighted the importance of nanoparticle ensembles to manipulate linear optical phenomena, ${ }^{10,11}$ these ensembles are also anticipated to open new avenues for tailoring nonlinear optical phenomena.

Oligomers, that is, assemblies of nanoparticles with similar or identical composition, are gaining significant interest. ${ }^{11}$ For one thing, oligomers possess excitation modes that are governed by their symmetry, which can be made to resemble that of naturally occurring molecules. ${ }^{12,13}$ This important feature makes oligomers excellent candidates for tuning optical responses. ${ }^{14,15}$ Furthermore, the versatility of configuring oligomers with different symmetries gives rise to Fano-like resonances that can be useful in sensing and spectroscopy. ${ }^{16}$ Similar to previous works that studied the correlation between molecular symmetries (e.g., $n$-fold rotationally symmetric molecules) and harmonics generation, ${ }^{17-19}$ emerging approaches have revealed that oligomers of varied spatial symmetries can be used as promising platforms for modifying nonlinear effects such as two-photon luminescence, ${ }^{20}$ secondharmonic generation (SHG), ${ }^{21-23}$ third-harmonic generation, ${ }^{24}$ and four-wave mixing. ${ }^{25}$ So far, these nonlinear effects have been investigated using either plane-wave or focused-beam excitation with homogeneous states of polarization.

An emerging way to explore the optical responses of nanoparticles is through the use of cylindrical vector beams $(\mathrm{CVBs})^{26}$ that exhibit inhomogeneous states of polarization. When CVBs are focused, they give rise to unique electric and magnetic field distributions in the focal volume. ${ }^{27,28}$ For example, focusing a radially polarized CVB leads to a longitudinal (out-of-plane) electric field component along the main direction of propagation and transverse (in-plane) electric field components that follow the radial distribution in the focal plane. On the other hand, the focusing of an azimuthally polarized CVB leads to purely transverse electric field

Received: January 22, 2018

Revised: $\quad$ March 20, 2018

Published: March 27, 2018 
components that follow the azimuthal distribution also in the focal plane. Because of these intriguing characteristics, CVBs are used as novel probes for imaging the orientation of molecules or nanostructures. ${ }^{29-33}$ Owing to the tensorial character of nonlinear interactions, CVBs are also used to improve the orientation sensitivity of nonlinear microscopy techniques. ${ }^{34-39}$ In the context of oligomer studies, focused CVBs have been shown to excite collective dark modes (with zero net dipole moment), which cannot be accessed using linear polarization under normal incidence. ${ }^{40-43}$ Using far-field scanning single-photon luminescence ${ }^{44}$ and near-field scanning optical microscopies, ${ }^{45,46}$ CVBs also show unique potential for studying oligomers with varied structural symmetries but the use of nonlinear excitation in this context is still unexplored.

Here, we demonstrate the use of SHG microscopy with CVBs for investigating individual plasmonic oligomers with different symmetries. The oligomers are prepared by electronbeam lithography and carefully designed to follow the transverse electric-field distributions of the respective focused CVBs. We find that SHG from plasmonic oligomers is significantly influenced by the CVB polarization and interparticle coupling effects. Our experimental results are in very good agreement with our calculations based on the frequency-domain boundary element method (BEM). Our work further highlights the potential of careful beam structuring and nanoparticle assembly for tailoring nonlinear optical effects on the nanoscale.

Our oligomers consist of gold nanorods ordered in radially or azimuthally symmetric configurations (Figure la,c,e,f). The
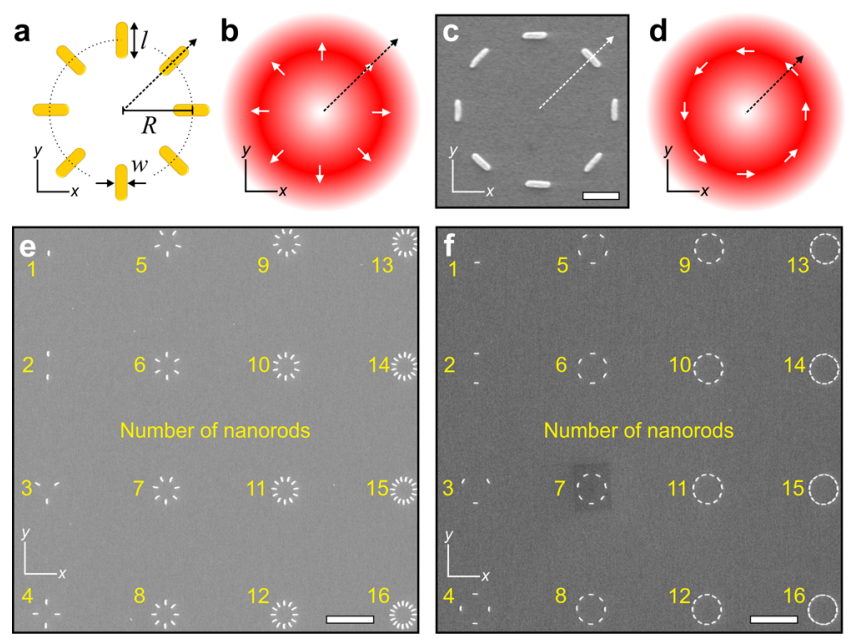

Figure 1. Configurations of oligomers and CVBs. (a) Schematic of an oligomer with radial configuration consisting of eight nanorods of width $w$ and length $l$ with their centers arranged on a circle of radius $R$. With respect to the radial vector from the center of the oligomer (dashed arrows), the (a) radial and (c) azimuthal oligomers are composed of nanorods with longitudinal axes that are oriented at $0^{\circ}$ and $90^{\circ}$, respectively. (c) Representative SEM image of an azimuthal oligomer consisting of eight nanorods. Scale bar: $250 \mathrm{~nm}$. (b,d) Sketches of matching radially and azimuthally polarized CVBs with different orientations of the transverse electric-field vectors in the focal plane (solid white arrows). With respect to the radial vector from the center of the laser beam focus (dashed arrows), the (b) radial and (d) azimuthal CVBs exhibit transverse electric field vectors that are oriented at $0^{\circ}$ and $90^{\circ}$, respectively. (e,f) Representative SEM images of arrays of (e) radial and (f) azimuthal oligomers with increasing numbers of nanorods (see marks). Scale bars: $2 \mu \mathrm{m}$. distance between the center of the oligomer and the center of a single nanorod is equal to the radius of the oligomer $R$. We used $R=485 \pm 5 \mathrm{~nm}$ to closely match the size of the oligomer to the size of the beam focus that is used in the nonlinear experiments. Each nanorod has a width $w$ and length $l$. We specifically used $w=46 \pm 5 \mathrm{~nm}$ and $l=165 \pm 5 \mathrm{~nm}$ so the longitudinal dipolar resonance of the nanorod is close to the fundamental wavelength of the laser $(1060 \mathrm{~nm})$ (Figure S1). Several arrays of oligomers with different numbers of constituent nanorods were fabricated by electron-beam lithography. The oligomers were fabricated on a cover glass with a $50 \mathrm{~nm}$ indium tin oxide (ITO) layer on top. A poly(methyl methacrylate) (PMMA) bilayer served as the electron-beam resist. After exposure, the resist was developed and treated by anisotropic oxygen plasma etching to remove potential residues within the nanopatterns. A $20 \mathrm{~nm}$ gold layer was deposited by thermal evaporation. Although the resulting thickness $(t)$ of the gold structures is comparable to the skin depth, this thickness is still much higher than the nonlocality length $\left(\sim 1 \mathrm{~nm}\right.$ in the optical regime). ${ }^{47}$ After a lift-off process, the sample fabrication was completed. The geometrical dimensions of the nanorods in the fabricated samples were independently confirmed by scanning-electron microscopy (SEM).

Two nanorod arrangements were investigated for maximum geometric overlap with the electric fields in the laser beam focus (Figure 1a,c). The orientation of a single rod is based on the direction of the local transverse electric field formed in the focus of the CVB. The direction of the longitudinal axis of single nanorods is derived from a cylindrical coordinate system, the origin of which is positioned at the center of the oligomer. For the radial oligomers, the longitudinal axis of the constituent nanorods is oriented parallel to the radial vector (Figure 1a). For azimuthal oligomers, the nanorods are oriented perpendicular to the radial vector (Figure 1c). Each fabricated array consist of 16 oligomers with $5 \mu \mathrm{m}$ separation with fixed oligomer and nanorod dimensions and with the number of nanorods $n$ per oligomer increasing from 1 to 16 . The SEM image of a representative array of radial (azimuthal) oligomers is shown in Figure 1e (Figure 1f). This layout allowed us to study individual oligomers that possess $n$-fold rotational symmetries in the focal plane. The distance between the neighboring nanorods of an oligomer decreases as the number of nanorods increases, and the degree of coupling between the neighboring nanorods of an oligomer is expected to increase correspondingly. Because of constraints in the lithographic fabrication, we limited the maximum number of nanorods in an oligomer to 16. Aside from this generic layout, we also prepared oligomers with identical features $(l=145 \pm 5 \mathrm{~nm}, w=40 \pm 5$ $\mathrm{nm}, t=20 \pm 5 \mathrm{~nm}, R=472 \pm 5 \mathrm{~nm})$ in a $2 \times 2$ layout. These oligomer samples were used in preliminary nonlinear optical experiments to prove that the observed signal is indeed dominated by SHG (Figure S2).

A custom-built point-scanning far-field nonlinear microscope was used to investigate the oligomers. ${ }^{48}$ This approach was chosen in order to address SHG from individual oligomers. The microscope uses a femtosecond laser (excitation wavelength of $1060 \mathrm{~nm}$, repetition rate of $80 \mathrm{MHz}$, pulse duration of $140 \mathrm{fs}$ ) as the excitation source. After performing beam collimation, expansion, and polarization manipulation (see below), the laser beam is directed toward a microscope objective [numerical aperture (NA) of 0.8 ]. The same objective is used to collect the nonlinear signals from the oligomers. Appropriate optical filters 
(fundamental wavelength block and SHG narrow bandpass at $531 \pm 11 \mathrm{~nm})$ and a cooled photomultiplier tube are used to discriminate and detect the second-harmonic wavelength around $530 \mathrm{~nm}$.

Two types of CVBs (focused radially and azimuthally polarized beams, from now on called radial and azimuthal CVBs) were synthesized and used in the experiments (Figure $1 \mathrm{~b}, \mathrm{~d}$ and S3). To generate radial and azimuthal CVBs, a polarization mode converter (Arcoptix S.A.) in tandem with a spatial filter is used. When this CVB is used as the input beam in our microscope, we achieve a transverse spot size of about 1 $\mu \mathrm{m}$ at the focal plane. This size corresponds to the distance between the maxima of the CVB at the focal plane. A computer-controlled three-axis piezo-scanning stage is used to position and translate the oligomer sample with respect to the stationary beam focus. The collection time for each sample position, that is, pixel, is $50 \mathrm{~ms}$. To create a scanning microscopy image, the SHG signal is collected pixel-by-pixel, that is, as a function of spatial coordinates $(x, y)$, and integrated as the sample is scanned across the focal plane. Since the SHG response is tensorial in nature, the resulting image is strongly dependent on the vectorial properties of the focused beam and not solely due to the convolution of the excitation point spread function and the geometry or size of the oligomer. ${ }^{39}$ Additional reference measurements are performed using linear polarizations of the excitation beams. To generate linear polarization of any orientation, a half-wave plate is used.

To compare the SHG signals from the oligomers, the imaging is always performed within a single array and the excitation power is maintained at $1 \mathrm{~mW}(0.2 \mathrm{~mW})$ for the different CVB (linear) polarizations. A lower input power is used for linear polarization in order to prevent sample damage. Throughout the paper, the term "symmetric illumination" will be used to denote the situation whenever the center of an oligomer and the center of the laser beam focus coincide; any other situations are denoted as "asymmetric illumination".

To gain more insight into the experimental SHG signals, we performed surface SHG modeling based on the frequencydomain BEM. ${ }^{35}$ The BEM is suited for our purposes because it allows the use of a focused beam as excitation source. Moreover, the BEM is efficient since only the surface of each nanorod needs to be discretized. Previously, we have used this approach to elucidate the origin of SHG from well-isolated metal $^{35,36}$ and semiconductor ${ }^{38}$ nano-objects under different focusing conditions. Here, we extended the method to address SHG from several nanoparticles (the oligomer) that are simultaneously illuminated within the laser beam focus. To account for the glass substrate, the oligomers were embedded in homogenous medium $\left(n_{\mathrm{m}}=1.45\right) .{ }^{49}$ Further details about the BEM modeling and parameters used in the paper are found in the Supporting Information.

We first verified the nonlinear signals from the individual oligomers using our microscope (Figure S2). The nonlinear signals from the oligomers were found to be present and significant only whenever the excitation laser was pulsed and the detection was performed in the range of the $\mathrm{SH}$ wavelength using a narrow bandpass filter centered at $531 \mathrm{~nm}$ with a bandwidth of $\pm 11 \mathrm{~nm}$ before the detector. Conversely, the nonlinear signals were found to be reduced by 3 orders of magnitude when a notch filter centered at $530 \mathrm{~nm}$ with a bandwidth of $\pm 20 \mathrm{~nm}$ was used instead, that is, the range of the $\mathrm{SH}$ signal was blocked. Furthermore, the nonlinear signals that were acquired with the SHG filter were found to exhibit a quadratic power dependence on the input power. These results also show that two-photon luminescence ${ }^{50}$ from gold is about 3 orders of magnitude weaker than SHG. Finally, the maximum SHG signals from the oligomers are about 2-3 orders of magnitude higher than the background signals (about 20 counts per $50 \mathrm{~ms}$ ) from the ITO-covered glass. This result suggests that the effect of optical nonlinearities of the ITOcovered substrate $^{51}$ is negligible in this work.

We then performed SHG microscopy on the array of oligomers with increasing number of nanorods $(l=165 \pm 5$ $\mathrm{nm}, w=46 \pm 5 \mathrm{~nm}, t=20 \pm 5 \mathrm{~nm}, R=485 \pm 5 \mathrm{~nm})$. Upon comparing SHG images from different oligomers under different incident CVBs, the importance of the combination of the beam and oligomer symmetries becomes apparent (Figures 2-6). In general, an individual nanorod is strongly
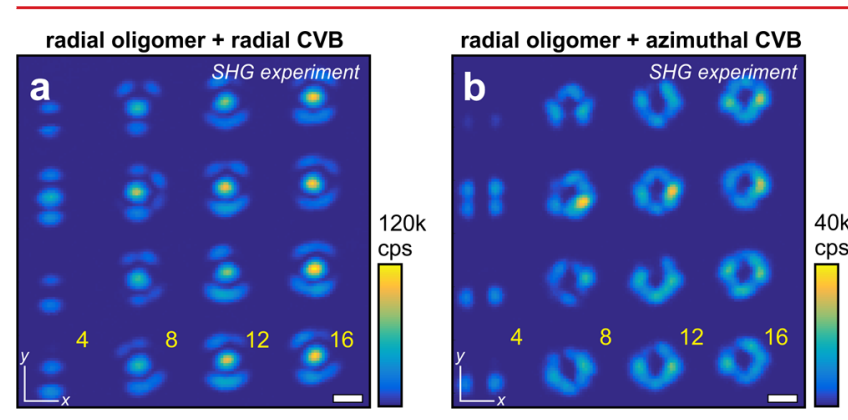

radial oligomer + radial CVB

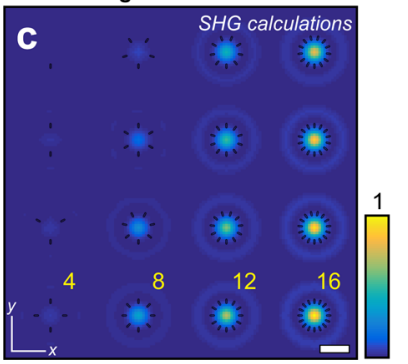

radial oligomer + azimuthal CVB

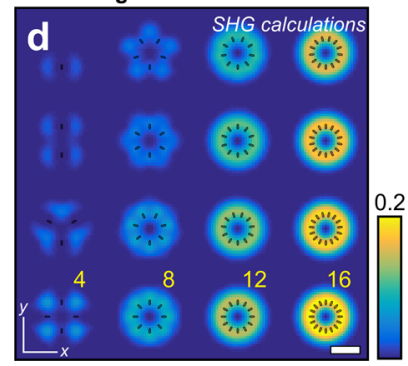

Figure 2. (a,b) Experimental SHG scanning microscopy images of the array of radial oligomers with increasing number of nanorods $(l=165$ $\pm 5 \mathrm{~nm}, w=46 \pm 5 \mathrm{~nm}, t=20 \pm 5 \mathrm{~nm}, R=485 \pm 5 \mathrm{~nm}$ ) using (a) radial and (b) azimuthal CVBs. The orientations of the oligomers and beams are the same as in Figure 1. The maximum SHG intensities are shown. Scale bar: $1 \mu \mathrm{m}$. (c,d) Calculated SHG scanning microscopy images (excitation wavelength of $1060 \mathrm{~nm}, \mathrm{NA}=0.8$ ) of the radial oligomers $(l=165 \mathrm{~nm}, w=46 \mathrm{~nm}, t=20 \mathrm{~nm}, R=485 \mathrm{~nm})$ with increasing number of nanorods using (c) radial and (d) azimuthal CVBs. The orientations of the oligomers (see marks) and beams are the same as in Figure 1. The SHG maps for each oligomer are simulated separately and then stitched together in the postprocessing. The maximum SHG intensities are shown. Scale bar: $1 \mu \mathrm{m}$.

excited at a given scan position when the local transversal electric field is parallel to the long axis of the nanorod. ${ }^{52}$ However, this is only valid whenever the nanorods behave as isolated nanoparticles. When two or more nanoparticles are situated close to each other forming nanoscale gaps, the nearfields of the individual nanoparticles form new collective plasmon resonances through plasmon hybridization. ${ }^{53}$ As we will show later, the SHG signals from such oligomers can be strongly modified by these collective modes that are supported by different oligomer arrangements.

The SHG images of the radial oligomers under excitation with radial (matching) and azimuthal (antimatching) CVBs are shown in Figure 2a,b. For the case of a radial CVB, the SHG 
scanning images of most oligomers exhibit a central intensity maximum surrounded by a ring of azimuthally varying intensity patterns (Figure 2a). Under symmetric illumination, the high SHG signal in the center arises from the simultaneous excitation of all the nanorods, that is, the local transverse electric fields of the radial CVB are simultaneously oriented parallel to the long axes of all the constituent nanorods. The azimuthally varying intensity patterns exhibit lower intensity levels than the central maximum and result when the oligomer is asymmetrically illuminated by the transverse electric fields of the radial CVB. Also, the resulting SHG scanning image patterns, intensities, and numbers of azimuthal maxima of the oligomers are found to strongly depend on the number of nanorods in the oligomer. The ring-like parts of the SHG scanning images exhibit the respective $n$-fold rotational symmetries, which is especially observable for the oligomers with a low number of nanorods. Maxima appear whenever the focus is centered outside the oligomer at the correct distance to excite an individual or few nanorods. These features, however, start to merge when the number of nanorods increases. For symmetric illumination, the overall SHG intensities in the central maximum increase with the number of nanorods. However, the SHG intensities at locations where the oligomer is asymmetrically illuminated by the $\mathrm{CVB}$ are more or less constant because only a fraction of the oligomer, which consists of a few nanorods, is illuminated by a section of the CVB.

In contrast, we always observe a central minimum surrounded by a ring of SHG maxima when radial oligomers are scanned through the focus of an azimuthal CVB (Figure 2b). Only low SHG intensity close to the background is observed when the radial oligomer is symmetrically illuminated. In this situation, the transverse electric fields of the azimuthal CVB are oriented perpendicular to each individual nanorod. In this case, the nanorods are not efficiently excited and contribute only weakly to the overall SHG. As for the case of the radial $\mathrm{CVB}$, the azimuthally varying ring-shaped maxima appear when the nanorods are asymmetrically illuminated by the transverse electric fields of the azimuthal CVB. Similar to the radial CVB results, the SHG scanning image patterns and intensities of the radial oligomer under azimuthal CVB excitation are influenced by the number of nanorods. As the transverse electric field components of the radial and azimuthal CVBs are oriented orthogonally to each other in the focal plane, the respective patterns within the outer rings of the SHG images for both CVBs are always rotated $90^{\circ}$ in-plane. This is most evident for oligomers with a low number of nanorods for the corresponding $n$-fold rotationally symmetric patterns.

The experimental results were found to be in good qualitative agreement with our SHG calculations based on BEM. The results of the calculations for the radial oligomers ( $l$ $=165 \mathrm{~nm}, w=46 \mathrm{~nm}, t=20 \mathrm{~nm}, R=485 \mathrm{~nm}$ ) under excitation with radial and azimuthal CVBs are shown in Figure $2 \mathrm{c}$,d. Under symmetric illumination with the matching (antimatching) CVB, the SHG scanning image patterns of the radial oligomers exhibit high (low) SHG intensities in the centers. Under asymmetric illumination, the SHG scanning images of the oligomers also form azimuthally modulated ring-like intensity distributions. Similar to the experimental results, the $n$-fold rotational symmetries of the oligomers with low numbers of rods are evident in the SHG scanning image patterns using both CVBs. Finally, the SHG intensities in the central maximum under excitation with the symmetrically illuminating

and matching radial CVB were found to continuously increase with the number of nanorods.

Analogous SHG experiments were then performed on the azimuthal oligomers using azimuthal (matching) and radial (antimatching) CVBs (Figure 3). Under excitation with an

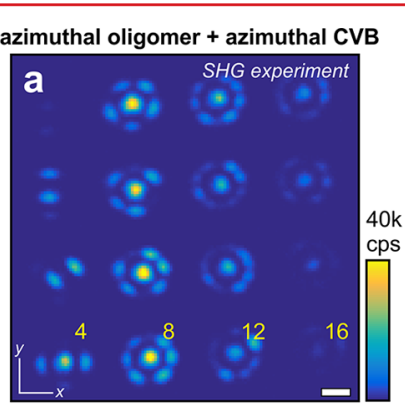

azimuthal oligomer + azimuthal CVB

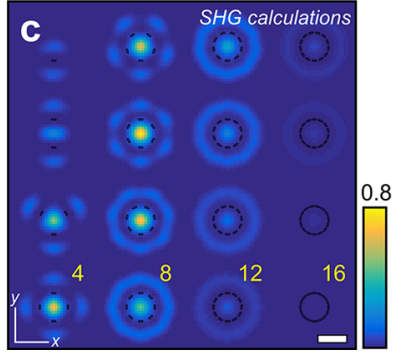

Figure 3. (a,b) Experimental SHG scanning microscopy images of the array of azimuthal oligomers with varying number of nanorods ( $l=$ $165 \pm 5 \mathrm{~nm}, w=46 \pm 5 \mathrm{~nm}, t=20 \pm 5 \mathrm{~nm}, R=485 \pm 5 \mathrm{~nm})$ using (a) azimuthal and (b) radial CVBs. The orientations of the oligomers and beams are the same as in Figure 1. The maximum SHG intensities are shown. Scale bar: $1 \mu \mathrm{m}$. (c,d) Calculated SHG scanning microscopy images $(\mathrm{NA}=0.8$, excitation wavelength of $1060 \mathrm{~nm}$ ) of the azimuthal oligomers $(l=165 \mathrm{~nm}, w=46 \mathrm{~nm}, t=20 \mathrm{~nm}, R=$ $485 \mathrm{~nm}$ ) with increasing number of nanorods under excitation with (c) azimuthal and (d) radial CVBs. The orientations of the oligomers (see marks) and beams are the same as in Figure 1. The SHG maps for each oligomer are simulated separately and then stitched together in the postprocessing. The maximum SHG intensities are shown. Scale bar: $1 \mu \mathrm{m}$.

azimuthal CVB, the experimental SHG scanning image pattern again exhibits a central intensity maximum surrounded by a ring of azimuthally varying intensity maxima (Figure 3a). Under symmetric illumination, the transverse electric fields of the azimuthal CVB are always oriented parallel to the long axis of each individual nanorod. All nanorods are simultaneously excited and contribute to the overall SHG intensity. On the other hand, asymmetric illumination results in azimuthally varying ring-shaped SHG intensity patterns with lower intensities than the central maximum. The resulting SHG patterns and intensities of the oligomers were also found to be strongly dependent on the number of nanorods in the oligomer. Again, the $n$-fold symmetry features for a low number of nanorods can be deduced from the SHG patterns. Most importantly, the overall SHG intensities from the oligomers were found to increase only up to the oligomer with seven nanorods. After that, the SHG intensities were found to strongly decrease. For example, the SHG intensity for the oligomer with 16 nanorods is comparable to or even weaker than that for a single nanorod.

When the azimuthal oligomer is symmetrically excited by the radial $\mathrm{CVB}$, a central SHG minimum close to the background 

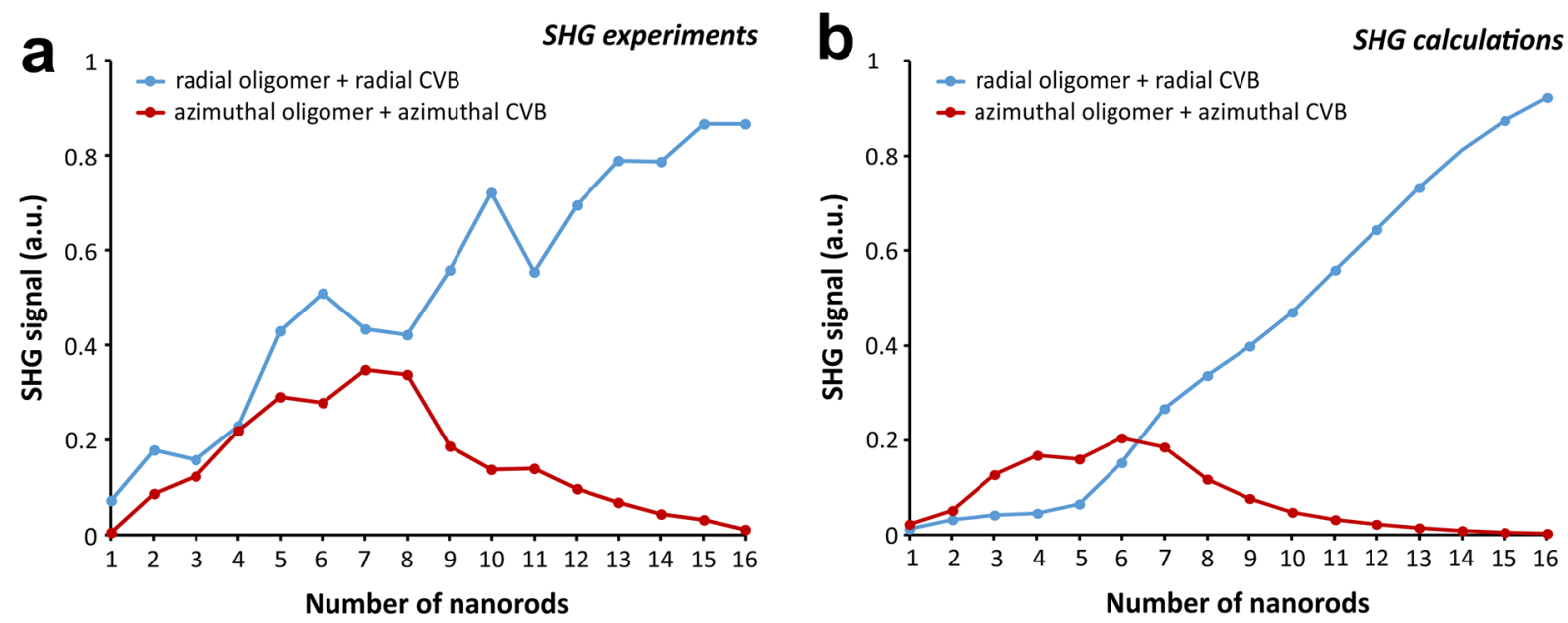

Figure 4. (a) Experimental and (b) calculated SHG signals from the radial and azimuthal oligomers $(l=165 \mathrm{~nm}, w=46 \mathrm{~nm}, t=20 \mathrm{~nm}, R=485 \mathrm{~nm})$ under the symmetrically illuminating and matching CVBs. The signals were taken from the center of the SHG images for each oligomer.

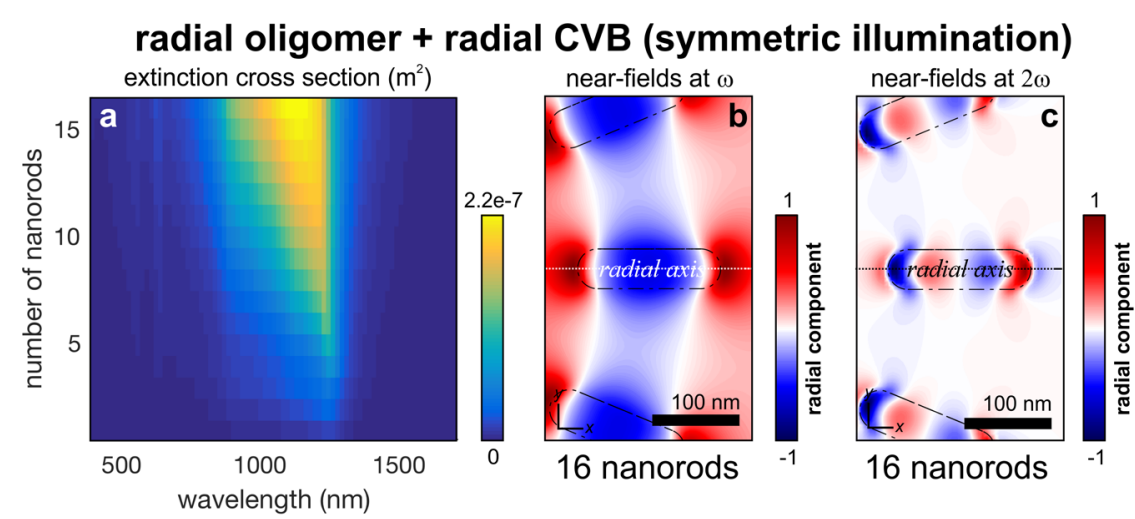

\section{radial oligomer + azimuthal CVB (symmetric illumination)}

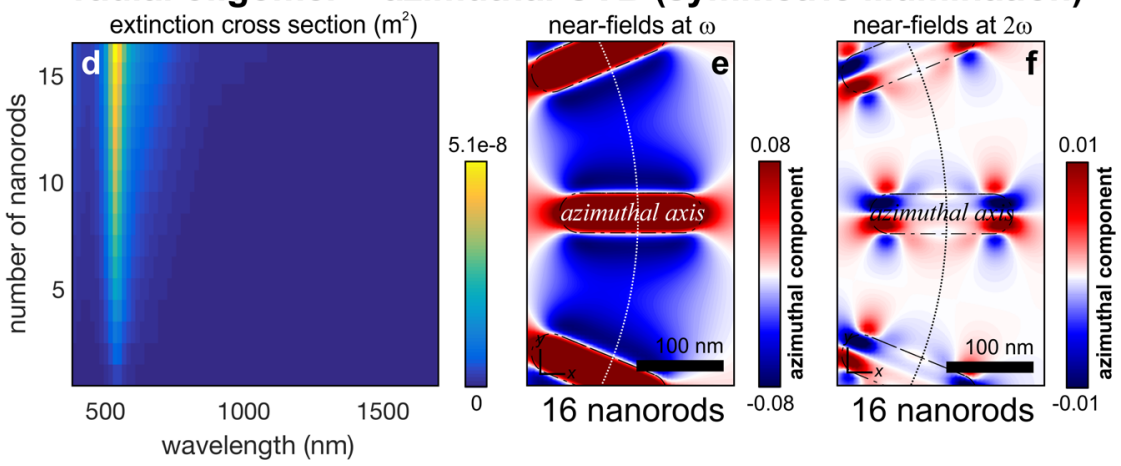

Figure 5. Calculated extinction spectra of radial oligomers $(l=165 \mathrm{~nm}, w=46 \mathrm{~nm}, t=20 \mathrm{~nm}, R=485 \mathrm{~nm})$ with varying number of nanorods $(1-$ 16) using focused (a) radial and (d) azimuthal CVBs under symmetric illumination. (b,c,e,f) Near-field distributions around a section of the radial oligomer with 16 nanorods in the transverse plane, that is, $20 \mathrm{~nm}$ above the mirror plane, (b,e) at the fundamental wavelength of $1060 \mathrm{~nm}$, and $(\mathrm{c}, \mathrm{f})$ at the second-harmonic wavelength of $530 \mathrm{~nm}$ for $(b, c)$ radial and $(e, f)$ azimuthal CVBs under symmetric illumination. In b,c (e,f), the real part of the radial $E_{\mathrm{r}}$ (azimuthal $E_{\phi}$ ) component of the total electric field is shown.

signal level is always observed since each individual nanorod is excited along the nonresonant short axis (Figure 3b). For asymmetric illumination, however, azimuthally varying SHG patterns appear with intensity levels and distributions that are again affected by the number of nanorods. The modulations of the ring patterns in the SHG images of the oligomers with a low number of nanorods are again complementary (rotated by $90^{\circ}$ ) to the azimuthal variation obtained with an azimuthal CVB. Similar to the azimuthal oligomer/azimuthal CVB results, the SHG intensities for azimuthal oligomers/radial CVB increase initially up to a certain number of nanorods ( 7 or 8 ) and then decrease, resulting in a faint ring-like image for the oligomer with 16 nanorods.

As can be seen in Figure 3, the SHG experimental results for the azimuthal oligomers were reproduced well by our SHG calculations ( $l=165 \mathrm{~nm}, w=46 \mathrm{~nm}, t=20 \mathrm{~nm}, R=485 \mathrm{~nm}$ ). As depicted in Figure $3 c, d$, the overall SHG intensities from the azimuthal oligomers clearly show an increase and subsequent decrease under both CVBs. At the same time, the maximum 
azimuthal oligomer + azimuthal CVB (symmetric illumination)

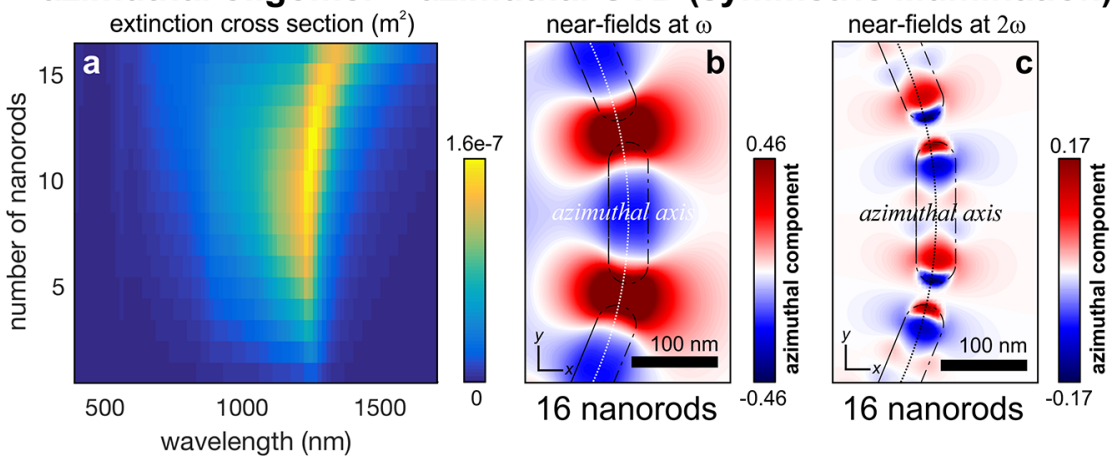

azimuthal oligomer + radial CVB (symmetric illumination)

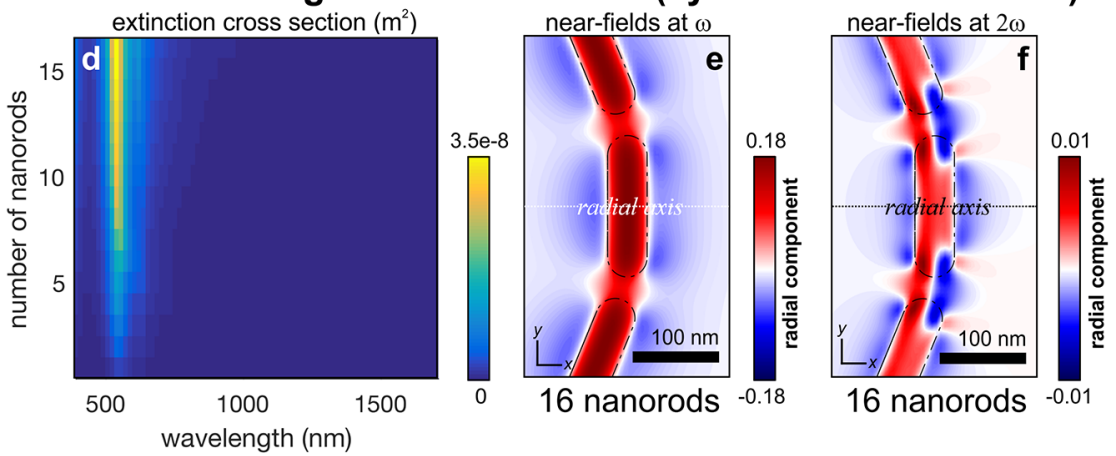

Figure 6. Calculated extinction spectra of azimuthal oligomers $(l=165 \mathrm{~nm}, w=46 \mathrm{~nm}, t=20 \mathrm{~nm}, R=485 \mathrm{~nm})$ with increasing number of nanorods (1-16) using focused (a) azimuthal and (d) radial CVBs under symmetric illumination. (b,c,e,f) Near-field distributions around a section of the azimuthal oligomer with 16 nanorods in the transverse plane, that is, $20 \mathrm{~nm}$ above the mirror plane, $(\mathrm{b}, \mathrm{e})$ at the fundamental wavelength of $1060 \mathrm{~nm}$ and $(c, f)$ at the second-harmonic wavelength of $530 \mathrm{~nm}$ for $(b, c)$ azimuthal and $(e, f)$ radial CVBs under symmetric illumination. In b,c $(e, f)$, the real part of the azimuthal $E_{\phi}\left(\right.$ radial $\left.E_{\mathrm{r}}\right)$ component of the total electric field is shown.

intensities that occur for the matching and nonmatching cases are comparable both experimentally and in simulations.

In general, we see a stark contrast in the behavior of the SHG signals between the radial and azimuthal oligomers under the corresponding matching CVBs. To further highlight the difference between these oligomers, we plotted the behavior of the SHG intensities at the center of the SHG patterns for the oligomers under the symmetrically illuminating and matching CVBs (Figure 4). For both experiment and calculations, we found that the SHG intensity from the radial oligomers increases approximately linearly with the number of nanorods. Furthermore, these results imply that the SHG from the radial oligomers is weakly affected by the interactions of the neighboring nanorods. In contrast, we found that the SHG intensity from the azimuthal oligomers increases and then decreases with the number of nanorods under the symmetrically illuminating and matching CVB. This behavior suggests that the interactions of the neighboring nanorods strongly influence the SHG from the whole oligomer. These results are reminiscent of earlier reports on the SHG behavior of plasmonic dimers with 15-90 nm-sized gaps that are illuminated by linearly polarized light. ${ }^{54}$ These studies concluded that interparticle coupling effects between the neighboring nanorods modify the overall SHG efficiency from the whole structure. Destructive interference between the out-of-phase near-fields were shown to lead to "silencing" of SHG in the far-field despite the existence of local field amplification in the gap.

We also note that small deviations between the calculated and experimental SHG images from the oligomers are evident.
For example, the experimental SHG images of the radial oligomers show a slight asymmetry along the $y$-axis. This could possibly originate from imperfections in the spatial structure of the radial CVB or from nanoscale variations of the fabricated oligomers. A systematic deviation for several structures points towards the CVB as the origin. To illustrate the dependence on structural defects, we calculated the SHG image of a radial oligomer with defects using a radial CVB with the same simulation parameters as those in Figure $2 \mathrm{c}$, and a surface mesh that corresponds to a radial oligomer that contains 9 nanorods instead of 16. As shown in Figure S4, the SHG image of this radial oligomer is affected by the quality of the whole structure even if the spatial structure of the radial CVB is ideal. Strong defects such as missing nanorods would be visible in the SEM images but similar effects may result from nanoscale variations in the nanorod geometries. This sensitivity is consistent with our earlier findings using SHG imaging of single nano-objects using CVBs. ${ }^{35}$

We further confirmed our results by performing SHG microscopy and calculations on the same oligomers $(l=165$ $\mathrm{nm}, w=46 \mathrm{~nm}, t=20 \mathrm{~nm}, R=485 \mathrm{~nm}$ ) using linearly polarized light (Figure S5). High-intensity spots are typically seen in the SHG images of each oligomer. These spots originate from nanorods that are aligned with the electric field of the linear polarization. Similar to the results using CVBs, the SHG signals from the radial oligomers increase, whereas the intensities from the azimuthal oligomers increase and then decrease with the number of nanorods. Again, these results are in agreement with previous SHG work on dimers. ${ }^{54}$ 
To elucidate the origin of the SHG intensities in the oligomers that are excited by the corresponding CVBs, we calculated the linear extinction cross section and associated near-fields at the fundamental and second-harmonic frequencies as a function of the number of nanorods in the oligomer (Supporting Information). We first show the results for the radial oligomers at the fundamental frequency (Figure 5). Compared to an isolated nanorod, the resonance wavelength slightly shifts to shorter wavelengths with increasing number of nanorods for symmetric illumination and matching CVB (Figure 5a). The slight increase in resonance frequency is attributed to enhanced repulsion between equal charges in the neighboring nanorods when illuminated by the matching CVB. This situation is similar to the resonance behavior of a dimer which is driven by an electric field that is perpendicular to the axis of the dimer. ${ }^{55-57}$ In contrast, a symmetrically illuminating, but antimatching CVB is only able to nonresonantly excite the short-wavelength plasmon that corresponds to the width of the nanorods (Figure 5d). In this case, the transverse electric fields of the antimatching CVB are always perpendicular to the nanorods.

The near-field maps for the radial oligomer with 16 nanorods under the symmetrically illuminating and matching CVB at the fundamental frequency reveal that the nanorods are excited in a dipole-like manner, where the dipoles oscillate in-phase along the radial vector (Figure $5 b$ ). In these examples, the strongest electric fields are always located near the ends of the nanorods resulting in weak interactions between the adjacent nanorods. Under the symmetrically illuminating and antimatching CVB, the nanorods are also excited like dipoles that are in-phase along the azimuthal vector (Figure 5e). In this case, the strongest electric fields are always located at the long sides of the nanorods, resulting again in weak interactions between the adjacent nanorods.

In contrast, the linear extinction maps of the azimuthal oligomers under the symmetrically illuminating and matching CVB reveal a characteristic redshift of the plasmon resonance peak of the azimuthal oligomer with increasing number of nanorods (Figure 6a). The resonance frequency decrease is associated with the enhancement of the attraction between opposite charges in the neighboring nanorods when illuminated by the local electric fields of the matching CVB. This situation is similar to a bonding behavior of a dimer driven by an electric field that is parallel to the dimer axis, pushing the peak to longer wavelengths. ${ }^{55-57}$ Also, the extinction features of the azimuthal oligomer are generally weaker (by about 25\%), and broader than those of the radial oligomer under the matching CVB. In contrast, a symmetrically illuminating and antimatching CVB is only able to excite the short-wavelength plasmon resonance that corresponds to the width of the nanorods for all oligomers as expected (Figure 6d).

Additionally, the near-field maps for the azimuthal oligomer with 16 nanorods under the symmetrically illuminating and matching CVB at the fundamental frequency show that the nanorods are excited in the manner of dipoles that oscillate inphase along the azimuthal vector (Figure $6 \mathrm{~b}$ ). The strongest electric fields are always found between nanorods suggesting the possibility of strong near-field interactions especially for the oligomer with 16 nanorods with a gap size of about $25 \mathrm{~nm}$. Under the symmetrically illuminating and antimatching CVB, the nanorods are also excited like dipoles that are in-phase along the radial vector (Figure 6e). In this case, the strongest electric fields are always situated at the sides of the nanorods, which results in weak interactions between the neighboring nanorods.

The extinction and near-field maps show striking differences between the behavior of the two types of oligomers when excited by the CVBs. We associate these differences with different collective interactions between the neighboring nanorods supported by each type of oligomer. These results are in agreement with previous studies on oligomers that are excited by CVBs. ${ }^{40,41,46}$ More importantly, these collective interactions significantly influence the efficiency of the SHG from the oligomer structure. ${ }^{21-23,58}$ This is perhaps better seen in the calculated near-field maps of the second-harmonic fields for the oligomers and symmetrically illuminating and matching CVBs.

For the radial oligomer with 16 nanorods, the near-field maps at the second-harmonic frequency show that the nanorods display higher-order plasmon resonances for both CVBs (Figure 5c,f) which is obviously different from the case observed at the fundamental frequency. By comparing the features of the calculated SHG near field maps for the isolated nanorod and the constituent nanorods of the 16-nanorod oligomer (Figure S6), we can associate SHG with quadrupolelike modes, which is a distinguishing feature of SHG from centrosymmetric nanoparticles. ${ }^{59}$ Here, the SHG is forbidden within the electric dipole approximation and the $\mathrm{SH}$ emission must contain contributions from higher order modes. For nanorods with a thickness that is comparable to the excitation wavelength, the phase variation of the fundamental wave across the thickness of the nanorod could also result in a dipole-like SHG along the axis of beam propagation. ${ }^{59}$ In addition, the strongest electric fields are located near the ends (sides) of the nanorods under the symmetrically illuminating and matching (antimatching) CVB. For the antimatching CVB, the far-field SHG contributions from the nanorods are negligible as expected (Figure 5f). Also, we did not see significant changes in the corresponding near-field maps of the single nanorod when the number of nanorods in the radial oligomer is increased (Figures S6 and S7). We attribute slight changes in the near-field maps to the slight shifting of the fundamental resonance peak of the oligomer. This further confirms that the neighboring nanorods of this oligomer remain weakly coupled. Hence, the SHG from the radial oligomers is not significantly modified by the collective excitation of its constituent nanorods.

For the azimuthal oligomer with 16 nanorods, the near-field maps at the second-harmonic frequency also show that the nanorods display higher-order plasmon resonances for both CVBs (Figure 6c,f). In addition, the strongest electric fields are located near the ends (sides) of the nanorods under the symmetrically illuminating and matching (antimatching) CVB. For the matching $\mathrm{CVB}$, the near-field maps indicate regions between the nanorods where the second-harmonic fields vanish (Figure 6c). In addition, we see significant changes in the corresponding near-field maps when the number of nanorods in the azimuthal oligomer is increased (Figure S6). For example, the near-field maps of the oligomers that consist of one up to seven or eight nanorods look similar and remain nearly unchanged. Beyond that number of nanorods, the nearfield strengths in the vicinity of a single nanorod are significantly decreased. Note that for the corresponding nearfield maps at the fundamental frequency, the near-field strengths are almost the same for different numbers of nanorods (Figure S7). This confirms that the collective 
interactions between the neighboring nanorods of this oligomer significantly influence the overall SHG from the oligomer. Here, we attribute the observed collective far-field effects to a combination of the significant shifts in the fundamental resonance peak of the oligomers, and the silencing of the SHG at the gaps of the neighboring nanorods, which already becomes manifest in the near-field simulations. Again, these results are in line with earlier reports on the SHG behavior of dimers that are illuminated by linearly polarized light. ${ }^{54}$ For the antimatching CVB, the far-field SHG contributions from the nanorods are negligible as expected (Figure $6 \mathrm{f}$ ). Finally, the corresponding near field maps for the orthogonal components of all these configurations revealed even more complicated patterns (Figures S8 and S9). The analysis of these data, however, goes beyond the scope of the present work.

To further elucidate the collective resonance effects from the oligomer, we performed additional extinction calculations using BEM. Here, we concentrated on using azimuthal oligomers that are symmetrically illuminated by an azimuthal CVB. We performed the BEM calculations using the same parameters as before. Starting from the configuration of the 16-nanorod oligomer with a fixed gap size, we decreased the number of rods one at a time (Figure S10). We found that the extinction features vary strongly with the number of interacting nanorods. Furthermore, the extinction features of the 16-nanorod oligomer are not the same as those of the corresponding systems with few nanorods, e.g., one up to eight. In fact, the dominant collective resonance peak for the 16-nanorod oligomer is different from the plane-wave-excited collective resonance peak of a linear chain of 16 nanorods of similar dimensions which are aligned end-to-end and with equivalent gap size. In the linear chain of nanoparticles, e.g., nanospheres ${ }^{60}$ or nanorods ${ }^{61}$ with narrow gaps, the dominant collective resonance peak, i.e., lowest energy mode, shifts toward longer wavelengths as the chain length, i.e., number of nanoparticles, increases. Clearly, the excitation of oligomers using CVBs brings a new aspect that goes beyond the results of plasmonic dimers and capabilities of conventional optical techniques that rely on plane-wave excitation for studying nonlinear optical interactions of coupled assemblies of nanoparticles.

Our results demonstrate the richness of the nonlinear optical properties of plasmonic oligomers that can be observed by using tailored light distributions. Although the main features of the results can be qualitatively understood by considering the SHG behavior of coupled plasmonic dimers with nanometric gaps, it is clear that additional work needs to be done in order to understand all the details of the various SHG signals. For example, the effects of Fano resonances using $\mathrm{CVBs}^{40}$ and asymmetric illumination ${ }^{62}$ are expected to play additional roles in the tailoring of nonlinear phenomena in oligomers. Such effects may be the subject of future experiments using intricate tailor-made nanorod arrangements. Another possible direction is to obtain experimental linear extinction measurements on the plasmonic oligomers using focused CVBs in support of the corresponding calculations. However, CVBs with broadband excitation remain a fundamental challenge in vector beam control and related disciplines. Nonetheless, we believe that our findings will generate interest in developing new types of polarization mode converters that are suitable for broadband excitation. One more interesting future work is the polarization analysis of the SHG signal for different combinations of oligomers and CVBs. However, the excitation of the sample with CVBs at the fundamental wavelength and the detection of the polarization of the SHG signal are mutually incompatible in the present reflection geometry as there are no optical components that transmit CVBs at one wavelength while they do not change polarization at another. Nevertheless, we believe that our findings will motivate interest in developing new types of polarization components, which are suitable for polarization analysis of CVBs.

It is also interesting to discuss the possibility of deducing the symmetry-related selection rules for SHG emission in our work. The symmetry rules have two different aspects. First, one needs to consider the intrinsic nonlinear response of the materials constituting the oligomers. Here we use the approach, which has been proven to work well in several important cases, ${ }^{7,63}$ where the nonlinearity arises from the surface response of the metal-dielectric interface. Such local response then needs to be integrated over multiple surfaces of the whole structure and is fully accounted for in our simulations. The other issue is whether certain types of overall symmetry rules could be established for the whole structure. This question is much more complicated because both the intrinsic material response and the experimental geometry have to be simultaneously considered. Although such selection rules have been established for isolated nanoparticles under plane-wave excitation ${ }^{59}$ or electric-field gradient, ${ }^{64}$ these rules do not apply for oligomers under focused CVB excitation, which exhibit varying states of polarization (SOP) at the focal volume. In fact, care has to be taken already when an isolated nanoparticle is subjected to focused linear or circular polarizations. ${ }^{65}$ In this case, subjecting a nanoparticle to regions in the focal volume with rapid spatial variations of field intensities ${ }^{65}$ or focused beams of arbitrary transverse mode structure ${ }^{66}$ can already influence the relative contribution of the high-order multipoles to the SHG process. Furthermore, in our experiments the sample is scanned under the focused beams and the symmetry of the experiment varies continuously. Hence, it is not possible to establish general symmetry rules for the overall experiment.

Nevertheless, our results support the increasing importance of developing new techniques to investigate collective effects for tailoring the nonlinear optical responses of complex plasmonic structures. While other works have highlighted the use of traditional polarizations (e.g., linear) to study nonlinear responses in oligomers, we have here emphasized the use of unconventional polarizations to investigate oligomers with matching spatial symmetries. This technique is expected to further improve the sensitivity of current nonlinear optical techniques for studying collective interactions and their impact on symmetry-sensitive optical phenomena such as SHG. ${ }^{21-23,58,67}$ Our study opens up new ways to couple inhomogeneous light fields into complex arrangements of nanoobjects, and to investigate and tailor nonlinear effects in optical nanocircuits ${ }^{68}$ using polarization control $^{39}$ in general. Alternatively, the oligomers can be used to benchmark the performance of imaging and spectroscopic techniques that rely on optical beams with tailorable states of polarization at the beam focus.

To summarize, we have introduced the use of SHG microscopy with CVBs to investigate plasmonic oligomers consisting of metal nanorods with systematically varied spatial symmetries. The oligomers were prepared by electron-beam lithography and carefully designed such that each nanorod exhibits a dipolar resonance near the excitation wavelength, and that the overall oligomer structure follows the transverse electric-field distributions of the focused CVBs. We observed 
that SHG efficiency from such oligomers is strongly dependent on the polarization of the incident CVB and the interparticle effects. Particularly strong SHG intensity is observed when oligomers are symmetrically excited with the matching CVB, such that all nanorods are simultaneously excited. The maximum SHG depends on an interplay between the increasing number of nanorods and their respective near-field interactions. We found very good qualitative agreement between our experimental results and calculations based on the frequencydomain boundary element method. This work opens new ways to address and tailor the second-harmonic response of plasmonic oligomers with high spatial symmetries.

\section{ASSOCIATED CONTENT}

\section{S Supporting Information}

The Supporting Information is available free of charge on the ACS Publications website at DOI: 10.1021/acs.nanolett.8b00308.

Additional experimental data and modeling results are provided (PDF)

\section{AUTHOR INFORMATION}

\section{Corresponding Authors}

*E-mail: godofredo.bautista@tut.fi.

*E-mail: monika.fleischer@uni-tuebingen.de.

ORCID

Godofredo Bautista: 0000-0003-0810-7139

\section{Author Contributions}

${ }^{\perp}$ G.B. and C.D. contributed equally to this work.

\section{Notes}

The authors declare no competing financial interest.

\section{ACKNOWLEDGMENTS}

This work was supported by grants of the Academy of Finland (267847 and 287651) and Tampere University of Technology (Investment funding for Optics and Photonics). Financial support by the European Cooperation in Science and Technology through COST Action MP1302 Nanospectroscopy (STSM) is gratefully acknowledged. We thank J. Mäkitalo for assistance in the BEM calculations.

\section{REFERENCES}

(1) Kauranen, M.; Zayats, A. Nat. Photonics 2012, 6, 737-748.

(2) Tuovinen, H.; Kauranen, M.; Jefimovs, K.; Vahimaa, P.; Vallius, T.; Turunen, J.; Tkachenko, N. V.; Lemmetyinen, H. J. Nonlinear Opt. Phys. Mater. 2002, 11 (4), 421-432.

(3) Wang, J.; Butet, J.; Baudrion, A.-L.; Horrer, A.; Lévêque, G.; Martin, O. J. F.; Meixner, A. J.; Fleischer, M.; Adam, P.-M.; Horneber, A.; Zhang, D. J. Phys. Chem. C 2016, 120, 17699-17710.

(4) Butet, J.; Duboisset, J.; Bachelier, G.; Russier-Antoine, I.; Benichou, E.; Jonin, C.; Brevet, P.-F. Nano Lett. 2010, 10, 1717-1721.

(5) Neacsu, C. C.; Reider, G. A.; Raschke, M. B. Phys. Rev. B: Condens. Matter Mater. Phys. 2005, 71, 201402.

(6) Mühlschlegel, P.; Eisler, H.-J.; Martin, O. J. F.; Hecht, B.; Pohl, D. W. Science 2005, 308 (5728), 1607-1609.

(7) Canfield, B. K.; Husu, H.; Laukkanen, J.; Bai, B.; Kuittinen, M.; Turunen, J.; Kauranen, M. Nano Lett. 2007, 7 (5), 1251-1255.

(8) Celebrano, M.; Wu, X.; Baselli, M.; Großmann, S.; Biagioni, P.; Locatelli, A.; De Angelis, C.; Cerullo, G.; Osellame, R.; Hecht, B.; Duò, L.; Ciccacci, F.; Finazzi, M. Nat. Nanotechnol. 2015, 10 (5), 412-417.

(9) Linnenbank, H.; Grynko, Y.; Förstner, J.; Linden, S. Light: Sci. Appl. 2016, 5, el6013.
(10) Fan, J. A.; Wu, C.; Bao, K.; Bao, J.; Bardhan, R.; Halas, N. J.; Manoharan, V. N.; Nordlander, P.; Shvets, G.; Capasso, F. Science 2010, 328 (5982), 1135-1138.

(11) Hentschel, M.; Saliba, M.; Vogelgesang, R; Giessen, H.; Alivisatos, A. P.; Liu, N. Nano Lett. 2010, 10 (7), 2721-2726.

(12) Chuntonov, L.; Haran, G. Nano Lett. 2011, 11 (6), 2440-2445.

(13) Brandl, D. W.; Mirin, N. A.; Nordlander, P. J. Phys. Chem. B 2006, 110, 12302-12310.

(14) Hentschel, M.; Dregely, D.; Vogelgesang, R.; Giessen, H.; Liu, N. ACS Nano 2011, 5 (3), 2042-2050.

(15) Hentschel, M.; Schäferling, M.; Weiss, T.; Liu, N.; Giessen, H. Nano Lett. 2012, 12 (5), 2542-2547.

(16) Luk'yanchuk, B.; Zheludev, N. I.; Maier, S. A.; Halas, N. J.; Nordlander, P.; Giessen, H.; Chong, C. T. Nat. Mater. 2010, 9 (9), 707-715.

(17) Eichmann, H.; Egbert, A.; Nolte, S.; Momma, C.; Wellegehausen, B.; Becker, W.; Long, S.; McIver, J. K. Phys. Rev. A: At., Mol., Opt. Phys. 1995, 51 (5), R3414.

(18) Baykusheva, D.; Ahsan, M. S.; Lin, N.; Wörner, H. J. Phys. Rev. Lett. 2016, 116, 123001.

(19) Reich, D. M.; Madsen, L. B. Phys. Rev. Lett. 2016, 117, 133902.

(20) Deng, H.-D.; Chen, X.-Y.; Xu, Y.; Miroshnichenko, A. E. Nanoscale 2015, 7 (48), 20405-20413.

(21) Thyagarajan, K.; Butet, J.; Martin, O. J. F. Nano Lett. 2013, 13, $1847-1851$.

(22) Grinblat, G.; Rahmani, M.; Cortes, E.; Comedi, D.; Maier, S. A.; Bragas, A.; et al. Nano Lett. 2014, 14, 6660-6665.

(23) Liu, S.; Sok, E.; Leong, P.; Li, G.; Hou, Y.; Deng, J.; Teng, J. H.; Ong, H. C.; Lei, D. Y. ACS Nano 2016, 10, 1442-1453.

(24) Shcherbakov, M. R.; Shorokhov, A. S.; Neshev, D. N.; Hopkins, B.; Staude, I.; Melik-Gaykazyan, E.; Ezhov, A. A.; Miroshnichenko, A. E.; Brener, I.; Fedyanin, A. A.; Kivshar, Y. S. ACS Photonics 2015, 2 (5), 578-582.

(25) Zhang, Y.; Wen, F.; Zhen, Y.-R.; Nordlander, P.; Halas, N. J. Proc. Natl. Acad. Sci. U. S. A. 2013, 110 (23), 9215-9219.

(26) Zhan, Q. Adv. Opt. Photonics 2009, 1 (1), 1-57.

(27) Youngworth, K. S.; Brown, T. Opt. Express 2000, 7 (2), 77-87.

(28) Dorn, R.; Quabis, S.; Leuchs, G. Phys. Rev. Lett. 2003, 91 (23), 233901.

(29) Novotny, L.; Beversluis, M. R.; Youngworth, K. S.; Brown, T. G. Phys. Rev. Lett. 2001, 86 (23), 5251-5254.

(30) Fleischer, M.; Stanciu, C.; Stade, F.; Stadler, J.; Braun, K.; Heeren, A.; Häffner, M.; Kern, D. P.; Meixner, A. J. Appl. Phys. Lett. 2008, 93, 111114.

(31) Failla, A. V.; Qian, H.; Qian, H.; Hartschuh, A.; Meixner, A. J. Nano Lett. 2006, 6 (7), 1374-1378.

(32) Fleischer, M.; Zhang, D.; Braun, K.; Jäger, S.; Ehlich, R.; Häffner, M.; Stanciu, C.; Hörber, J. K. H.; Meixner, A. J.; Kern, D. P. Nanotechnology 2010, 21 (6), 065301.

(33) Meixner, A. J.; Jäger, R.; Jäger, S.; Bräuer, A.; Scherzinger, K.; Fulmes, J.; zur Oven Krockhaus, S.; Gollmer, D. A.; Kern, D. P.; Fleischer, M. Faraday Discuss. 2015, 184, 321-337.

(34) Yoshiki, K.; Hashimoto, M.; Araki, T. Jpn. J. Appl. Phys. 2005, 44 (34), L1066-L1068.

(35) Bautista, G.; Huttunen, M. J.; Mäkitalo, J.; Kontio, J. M.; Simonen, J.; Kauranen, M. Nano Lett. 2012, 12 (6), 3207-3212.

(36) Huttunen, M. J.; Lindfors, K.; Andriano, D.; Mäkitalo, J.; Bautista, G.; Lippitz, M.; Kauranen, M. Opt. Lett. 2014, 39 (12), 3686-3689.

(37) Reichenbach, P.; Horneber, A.; Gollmer, D. A.; Hille, A.; Mihaljevic, J.; Schäfer, C.; Kern, D. P.; Meixner, A. J.; Zhang, D.; Fleischer, M.; Eng, L. M. Opt. Express 2014, 22 (13), 15484-15501.

(38) Bautista, G.; Mäkitalo, J.; Chen, Y.; Dhaka, V.; Grasso, M.; Karvonen, L.; Jiang, H.; Huttunen, M. J.; Huhtio, T.; Lipsanen, H.; Kauranen, M. Nano Lett. 2015, 15, 1564-1569.

(39) Bautista, G.; Kauranen, M. ACS Photonics 2016, 3 (8), 13511370.

(40) Sancho-Parramon, J.; Bosch, S. ACS Nano 2012, 6 (9), 84158423. 
(41) Gómez, D. E.; Teo, Z. Q.; Altissimo, M.; Davis, T. J.; Earl, S.; Roberts, A. Nano Lett. 2013, 13, 3722-3728.

(42) Bao, Y.; Hu, Z.; Li, Z.; Zhu, X.; Fang, Z. Small 2015, 11, 21772181.

(43) Bao, Y.; Zhu, X.; Fang, Z. Sci. Rep. 2015, 5, 11793.

(44) Hentschel, M.; Dorfmüller, J.; Giessen, H.; Jäger, S.; Kern, A. M.; Braun, K.; Zhang, D.; Meixner, A. J. Beilstein J. Nanotechnol. 2013, $4,57-65$.

(45) Jäger, S.; Kern, A. M.; Hentschel, M.; Jäger, R.; Braun, K.; Zhang, D.; Giessen, H.; Meixner, A. J. Nano Lett. 2013, 13, 35663570.

(46) Yanai, A.; Grajower, M.; Lerman, G. M.; Hentschel, M.; Giessen, H.; Levy, U. ACS Nano 2014, 8 (5), 4969-4974.

(47) Stockman, M. I. Opt. Express 2011, 19 (22), 22029-22105.

(48) Turquet, L.; Kakko, J.-P.; Zang, X.; Naskali, L.; Karvonen, L.; Jiang, H.; Huhtio, T.; Kauppinen, E.; Lipsanen, H.; Kauranen, M.; Bautista, G. Laser Photonics Rev. 2017, 11 (1), 1600175.

(49) Knight, M. W.; Wu, Y.; Lassiter, J. B.; Nordlander, P.; Halas, N. J. Nano Lett. 2009, 9 (5), 2188-2192.

(50) Ghenuche, P.; Cherukulappurath, S.; Taminiau, T. H.; van Hulst, N. F.; Quidant, R. Phys. Rev. Lett. 2008, 101, 116805.

(51) Alam, M. Z.; De Leon, I.; Boyd, R. W. Science 2016, 352 (6287), 795-797.

(52) Hubert, C.; Billot, L.; Adam, P.-M.; Bachelot, R.; Royer, P.; Grand, J.; Gindre, D.; Dorkenoo, K. D.; Fort, A. Appl. Phys. Lett. 2007, 90 (18), 181105.

(53) Prodan, E.; Radloff, C.; Halas, N. J.; Nordlander, P. Science 2003, 302 (5644), 419-422.

(54) Berthelot, J.; Bachelier, G.; Song, M.; Rai, P.; Colas des Francs, G.; Dereux, A.; Bouhelier, A. Opt. Express 2012, 20 (10), 1049810508 .

(55) Rechberger, W.; Hohenau, A.; Leitner, A.; Krenn, J. R.; Lamprecht, B.; Aussenegg, F. R. Opt. Commun. 2003, 220 (1-3), 137-141.

(56) Aizpurua, J.; Bryant, G. W.; Richter, L. J.; García De Abajo, F. J.; Kelley, B. K.; Mallouk, T. Phys. Rev. B: Condens. Matter Mater. Phys. 2005, 71, 235420.

(57) Tabor, C.; Van Haute, D.; El-Sayed, M. A. ACS Nano 2009, 3

(11), 3670-3678.

(58) Rahmani, M.; Shorokhov, A. S.; Hopkins, B.; Miroshnichenko, A. E.; Shcherbakov, M. R; Camacho-Morales, R.; Fedyanin, A. A.; Neshev, D. N.; Kivshar, Y. S. ACS Photonics 2017, 4, 454.

(59) Dadap, J. I.; Shan, J.; Eisenthal, K. B.; Heinz, T. F. Phys. Rev. Lett. 1999, 83 (20), 4045-4048.

(60) Harris, N.; Arnold, M. D.; Blaber, M. G.; Ford, M. J. J. Phys. Chem. C 2009, 113 (7), 2784-2791.

(61) Jones, S. T.; Taylor, R. W.; Esteban, R.; Abo-Hamed, E. K.; Bomans, P. H. H.; Sommerdijk, N. A. J. M.; Aizpurua, J.; Baumberg, J. J.; Scherman, O. A. Small 2014, 10 (21), 4298-4303.

(62) Huang, J.-S.; Kern, J.; Geisler, P.; Weinmann, P.; Kamp, M.; Forchel, A.; Biagioni, P.; Hecht, B. Nano Lett. 2010, 10, 2105-2110.

(63) Butet, J.; Thyagarajan, K.; Martin, O. J. F. Nano Lett. 2013, 13 (4), 1787-1792.

(64) Brudny, V. L.; Mendoza, B. S.; Luis Monchán, W. Phys. Rev. B: Condens. Matter Mater. Phys. 2000, 62, 11152.

(65) Finazzi, M.; Biagioni, P.; Celebrano, M.; Duò, L. Phys. Rev. B: Condens. Matter Mater. Phys. 2007, 76, 125414.

(66) Bethune, D. S. Opt. Lett. 1981, 6 (6), 287-289.

(67) Gennaro, S. D.; Rahmani, M.; Giannini, V.; Aouani, H.; Sidiropoulos, T.; Cia, M. N.; Maier, S. A.; Oulton, R. F. Nano Lett. 2016, 16, 5278-5285.

(68) Engheta, N. Science 2007, 317 (5845), 1698-1702. 